\title{
Noise in graphene and carbon nanotube devices
}

\author{
Giuseppe Iannaccone, Alessandro Betti, Gianluca Fiori \\ Dipartimento di Ingegneria dell'Informazione: Elettronica Informatica, Telecomunicazioni \\ University of Pisa, Via G. Caruso 16, I-56126, Pisa, Italy \\ g.iannaccone@unipi.it
}

\begin{abstract}
We discuss the shot noise properties of carbon-based transistors in which the channel is laterally confined, either in the form of graphene nanoribbons or of carbon nanotubes. We show with an simple compact model and with computationallyintensive statistical simulations that electron-electron interaction can lead to a significant suppression of shot noise, often overlooked when the device is described with the LandauerButtiker formalism. Finally, we show that interband tunneling can play a significant role in enhancing shot noise due to exchange of holes between drain and channel, that is a peculiar effect observable in the case of channel materials with very small energy gaps.
\end{abstract}

Keywords- Shot noise, graphene, carbon nanotubes, field-effect transistors

\section{INTRODUCTION}

Carbon-based electronics represents a vibrant research field since more than a decade [1]. It has been recently further ignited by the isolation of graphene in 2004 and by the promising experiments of electron transport. Indeed, suspended graphene exhibits mobility in excess of $10^{5} \mathrm{~cm}^{2} / \mathrm{Vs}$ at room temperature[2], attracting the interest of electron device researchers. However, graphene is a semimetal, and therefore hardly suitable as a channel material for transistors. Among different proposed techniques to induce a gap in graphene, lateral confinement is certainly seriously considered. It means either using Carbon NanoTubes (CNTs), or cutting graphene in narrow stripes, the so-called Graphene NanoRibbons (GNRs). Several theoretical and experimental investigations of FETs based carbon nanotubes or nanoribbons have been documented in the literature.

The interest in noise in graphene sheets and quasi-one dimensional carbon conductors is more due to the fundamental aspects of electron transport it can reveal, than to the practical implications on device performance, given the premature stage of carbon electronics technology. Tworzydło et al. [3] have demonstrated that at zero temperature, with the Fermi energy at the Dirac point, ballistic graphene exhibits a shot noise Fano factor of $1 / 3$. The Fano factor $F=S /(2 q I)$, i.e. the ratio of the current noise power spectral density $S$ to the power spectral density of a purely Poissonian process, $2 q I$, where $q$ is the elementary charge and $I$ is the DC current in the channel. Experiments have substantially confirmed this prediction [4][5].

For carbon-based materials more interesting for device applications, such as carbon nanotubes and graphene nanoribbons, most published works focus on 1/f noise [6][7]. In both cases they find that at least up to $100 \mathrm{~Hz}$, noise is dominated by $1 / f$ noise in the range of typical current bias of interest. In our opinion, these results are extremely interesting, but are more an indication of the improvements required by fabrication technology than of the noise properties of mature carbon-based electron devices. $\mathrm{Wu}$ et al. [8] report suppression and enhancement of shot noise in multi-walled CNT FETs, at $4.2 \mathrm{~K}$.

Following these considerations, in this manuscript we will primarily focus on the shot noise properties of laterally confined carbon-based transistors, such as quasi onedimensional channels based on graphene nanoribbons or on carbon nanotubes. Most of the properties we shall discuss are not strictly limited to graphene, and extend more generally to quasi one-dimensional transistors.

However, carbon-based FET channels have some specificities. First, they can have a very small gap. The energy gap is roughly inversely proportional to width or diameter, and as a rule of thumb the transversal dimension has to be about $1 \mathrm{~nm}$ to induce a gap of order $1 \mathrm{eV}$. This means that, in practical cases, carbon-based channels can have energy gaps of $100 \mathrm{meV}$ or smaller. Second, electrons and holes have symmetric dispersion relations with small effective mass. Both aspects imply a potential significant role for interband tunneling.

In the following, we first examine shot noise in GNR and CNT FETs with an analytical model, and then with detailed physical simulations.

\section{ANALYTICAL MODEL}

An analytical model of shot noise in nanoscale FETs with two-dimensional channel is reported in [9]. We can follow the same derivation for one-dimensional nFETs (not shown here), including also the possibility of hole injection from the drain. We assume that hole injection from the source is inhibited at the bias we consider (weak or strong inversion).

The DC current and the low-frequency power spectral density of shot noise read, respectively:

$$
I=q \int_{0}^{\infty} d E N_{1 D} v_{z}\left(f_{S}-f_{D}\right)
$$

and

$$
\begin{aligned}
S(0) & =2 q^{2} \int_{0}^{\infty} d E N_{1 D} v_{z}[1-g(E)]^{2} f_{S}\left(1-f_{S}\right) \\
& +2 q^{2} \int_{0}^{\infty} d E N_{1 D} v_{z}[1-g(E)]^{2} f_{D}\left(1-f_{D}\right) \\
& +2 q^{2} \int_{0}^{\infty} d E N_{1 D}^{h} v_{z} g(E)^{2} f_{D p}\left(1-f_{D p}\right)
\end{aligned}
$$

where 


$$
g(E)=\frac{1}{v_{z}} \frac{v_{S} C_{n S}-v_{D} C_{n D}}{C_{T}+C_{n S}+C_{n D}+C_{p D}},
$$

$E$ is the kinetic energy, $N_{I D}$ is the one-dimensional density of states in the channel, $f_{S}$ and $f_{D}$ are the Fermi-Dirac occupation factors at source and drain, respectively, $v_{z}=\sqrt{2 E / m}$ is the velocity in the longitudinal direction for a free electron gas. $C_{T}$ is the total geometrical capacitance as seen by the channel, $C_{n S}$ and $C_{n D}$ are the quantum capacitances associated to electron charge injected by the source or drain, respectively, $C_{p D}$ is the quantum capacitance for holes injected by the drain. Finally, $v_{S}$ and $v_{D}$ are weighted averages of electron velocity.

We assume that transmission probability is 1 if electrons have energy larger than the potential energy peak in the barrier and 0 otherwise. We also assume that interband tunneling is so effective that holes in the channel are practically in equilibrium with the drain reservoir.

From the above expression of $S$ one can easily see that deviations from the poissonian formula $S=2 q I$ come from three sources:

- The first source of noise suppression is due to the factors $(1-f)$ in the first two terms on the right-hand side, and is due to the fermionic nature of carriers.

- The second source of noise suppression is the factor $[1-g(E)]^{2}$ in the first two terms of the right-hand side, which is due to electron-electron interaction, i.e. Coulomb repulsion.

- The third source provides enhancement of shot noise, and is due to the exchange of holes between the channel and the drain.

Of the three sources, often only the first is considered in the treatment of shot noise, following Landauer-Buttiker treatment of noise for conductors in quasi-equilibrium. However, it is clear that the other two factors, depending on the device and on the bias, can lead to further suppression, or even to enhancement of shot noise.

To examine in detail examples of these two cases, we use detailed physical simulations of CNT FETs. Results are valid also for GNR FETs with channel of comparable energy gap.

\section{COMPUTATIONAL APPROACH}

\section{A. Device simulation tool}

Our detailed physical simulations are based on the 3D device simulator NanoTCAD ViDES developed in Pisa [10]. It solves self-consistently the three-dimensional Poisson equation and the Schroedinger equation with open boundary conditions. The former reads

$$
\nabla[\epsilon(\vec{r}) \nabla \phi(\vec{r})]=-q\left[p(\vec{r})-n(\vec{r})+N_{D}^{+}(\vec{r})-N_{A}^{-}(\vec{r})+\rho_{f i x}\right]
$$

where $\phi$ is the electrostatic potential, $\varepsilon$ is the dielectric constant, $N_{D}{ }^{+}$and $N_{A}^{-}$are the concentration of the ionized donors and acceptors, respectively, $\rho_{f i x}$ is the fixed charge. Electrons and holes concentrations $n$ and $p$, respectively, are computed with the NEGF formalism, using a tight-binding Hamiltonian with an atomistic ( $\mathrm{p}_{\mathrm{z}}$ orbitals) real space basis set with a hopping parameter $t=2.7 \mathrm{eV}$. The Green's function can then be expressed as

$$
G(E)=\left[E I-H-\Sigma_{S}-\Sigma_{D}\right]^{-1}
$$

where $E$ now is the total energy, $I$ the identity matrix, $H$ the Hamiltonian, and $\Sigma_{S}$ and $\Sigma_{D}$ are the self-energies of the source and drain, respectively.

A point charge approximation is assumed, i.e. all the free charge around each carbon atoms is spread with a uniform concentration in the elementary cell including the atom. In particular, the electron and hole densities are computed from the Density of States (DOS), derived through the NEGF formalism. Assuming that the electrochemical potentials of the reservoirs are aligned at the equilibrium with the flat Fermi level of the CNT, and given that there are no fully confined states, we have

$$
\begin{aligned}
n(\vec{r})= & 2 \int_{E_{i}}^{+\infty} d E\left[\operatorname{DOS}_{\mathrm{S}}(\mathrm{E}, \overrightarrow{\mathrm{r}}) f\left(E-E_{F_{S}}\right)\right. \\
& \left.+\operatorname{DOS}_{\mathrm{D}}(\mathrm{E}, \overrightarrow{\mathrm{r}}) f\left(E-E_{F_{D}}\right)\right],
\end{aligned}
$$

and

$$
\begin{aligned}
p(\vec{r})= & 2 \int_{-\infty}^{E_{i}} d E\left\{\operatorname{DOS}_{\mathrm{S}}(\mathrm{E}, \overrightarrow{\mathrm{r}})\left[1-f\left(E-E_{F_{S}}\right)\right]\right. \\
& \left.+\operatorname{DOS}_{\mathrm{D}}(\mathrm{E}, \overrightarrow{\mathrm{r}})\left[1-f\left(E-E_{F_{D}}\right)\right]\right\}
\end{aligned}
$$

where $f$ is the Fermi-Dirac occupation factor, $\operatorname{DOS}_{S}\left(D O S_{D}\right)$ is the density of states injected by the source (drain), and $E_{F S}$ $\left(E_{F D}\right)$ is the Fermi level of the source (drain). The current is expressed by means of the Landauer formula. Particular attention must be put in the definition of each self-energy matrix: in our case we have considered a self-energy for semiinfinite leads as boundary condition for the Schrödinger equation.

\section{B. Shot noise computation including electron-electron interaction}

We have developed a theory that enables us to compute shot noise through statistical simulations of randomly injected electrons from the reservoirs, described in detail in [11] and [12]. The main advantage of our approach is the capability to fully include electron-electron interaction, both through Pauli exclusion, and through the Coulomb force.

Basically, we consider random statistical injection from the contacts. For electron and hole states injected by the reservoirs we consider a random occupation number $\sigma_{\alpha \mathrm{n}}(\mathrm{E})$ ( $\alpha=$ Source,Drain) that is 0 or 1 and - on average - corresponds to the Fermi occupation factor, i.e.,

$$
<\sigma_{\alpha n}(E)>=f_{\alpha}(E)
$$

Therefore we discretize the energy range of interest with a uniform mesh. For each energy bin, we extract a random number uniformly distributed between 0 and 1 . If the number is smaller then the Fermi factor, the occupation number is 1, otherwise it is 0 . Therefore, we have a random occupation function for electrons injected from the source and from the drain, and we solve NEGF and electrostatics self consistently. 
We repeat this process for an ensemble of occurrences, for which we collect total currents, transmission and reflection matrices.

The original formula by Landauer and Buttiker for noise in mesoscopic devices does not included coulomb repulsion between carriers [13][14]. We have derived an expression based on statistical averages to extend it to electrostatic interaction between carriers, therefore including the effect of potential energy fluctuations in the device. The reader is referred to [11] and [12] for a derivation based on second quantization theory, or to [16] for an alternative derivation.

Here, we just want to underline that noise is computed by computing statistical averages of expressions including transmission and reflection matrices obtained for each occurrence of the random occupation function. The average current is:

$$
\langle I\rangle=\frac{e}{h} \int d E\left\{\sum_{n \in S}\left\langle\left[\mathbf{t}^{\dagger} \mathbf{t}\right]_{n n} \sigma_{S n}\right\rangle_{s}-\sum_{k \in D}\left\langle\left[\mathbf{t}^{\prime \dagger} \mathbf{t}^{\prime}\right]_{k k} \sigma_{D k}\right\rangle_{s}\right\}
$$

whereas the current noise spectral density is

$$
\begin{aligned}
S(0)= & \left(\frac{e^{2}}{\pi \hbar}\right) \int d E \sum_{\alpha=S, D} \sum_{l \in \alpha}\left\langle[\tilde{\mathbf{t}}]_{\alpha, l l}\left(1-[\tilde{\mathbf{t}}]_{\alpha ; l l}\right) \sigma_{\alpha l}\right\rangle_{s} \\
& -\left(\frac{e^{2}}{\pi \hbar}\right) \int d E \sum_{\alpha=S, D} \sum_{\substack{l, p \in \alpha \\
l \neq p}}\left\langle[\tilde{\mathbf{t}}]_{\alpha ; l p}[\tilde{\mathbf{t}}]_{\alpha ; p l} \sigma_{\alpha l} \sigma_{\alpha p}\right\rangle_{s} \\
& -2\left(\frac{e^{2}}{\pi \hbar}\right) \int d E \sum_{k \in D} \sum_{p \in S}\left\langle\left[\mathbf{t}^{\prime} \mathbf{r}\right]_{k p}\left[\mathbf{r}^{\dagger} \mathbf{t}^{\prime}\right]_{p k} \sigma_{D k} \sigma_{S p}\right\rangle_{s} \\
& +\frac{4 \pi \hbar}{\Delta E} \operatorname{var}\left\{\frac{e}{h} \int d E\left(\sum_{n \in S}[\tilde{\mathbf{t}}]_{S ; n n} \sigma_{S n}-\sum_{k \in D}[\tilde{\mathbf{t}}]_{D ; k k} \sigma_{D k}\right)\right\}
\end{aligned}
$$

where $\hbar$ is the reduced Planck constant, $\Delta E$ is the energy step, the terms $\mathbf{t}$ and $\mathbf{r}$ refer to transmission and reflections matrices (details of their definitions in [11] and [12])

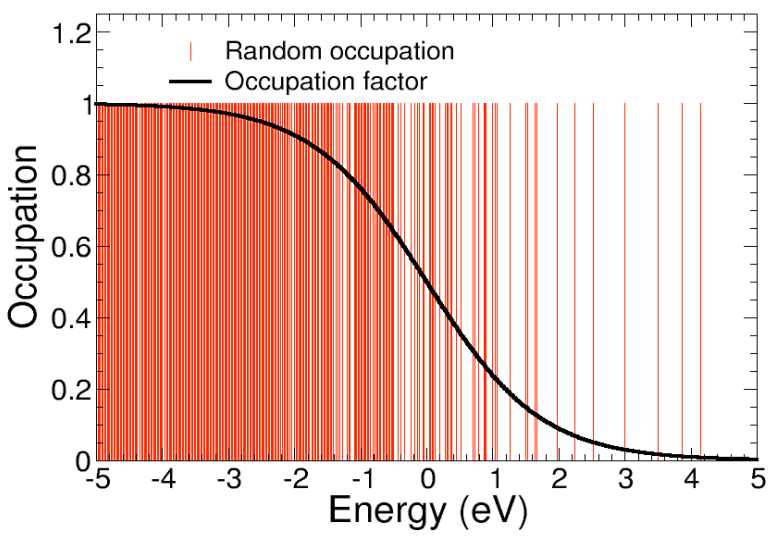

Figure 1. Example of random occupation number compared with occupation factor for energy bins of $0.5 \mathrm{meV}$.

The approach is general and can be applied to any ballistic conductor. We consider two different CNTs for the channel. One is a zig-zag $(25,0) \mathrm{CNT}$ with a band gap $E_{\text {gap }}=0.39 \mathrm{eV}$ and a diameter of $2 \mathrm{~nm}$. The oxide thickness is $1 \mathrm{~nm}$, the channel is undoped and has a length $L$ of $10 \mathrm{~nm}$.
Source and drain extensions have a length of $10 \mathrm{~nm}$ and are doped with a molar fraction $f=5 \times 10^{-3}$. The second is a $(13,0)$ CNT-FET, with $E_{\text {gap }}=0.75 \mathrm{eV}$, same geometry and doping profile.

The Fano factor $F$ is plotted as a function of gate overdrive for a $(25,0)$ and a $(13,0)$ zigzag CNT in Figs $2 \mathrm{a}$ and $2 b$.

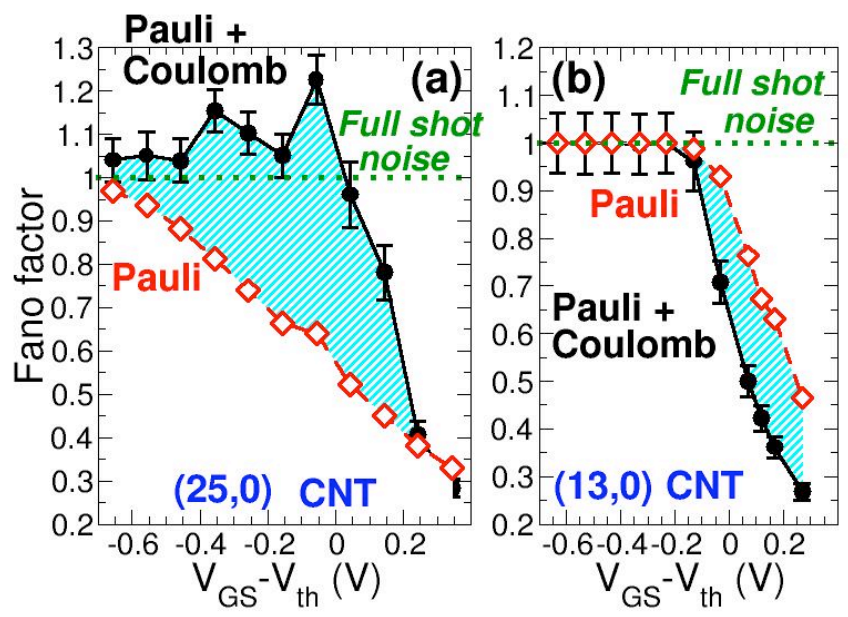

Figure 2. a) Fano factor as a function of the gate overdrive for a $(25,0)$ and b) $(13,0)$ CNT-FETs for $V_{\mathrm{DS}}=0.5 \mathrm{~V}$. The threshold voltage $V_{t h}$ is $0.43 \mathrm{~V}$ for the $(13,0) \mathrm{CNT}-\mathrm{FET}$, and $0.36 \mathrm{~V}$ for the $(25,0) \mathrm{CNT}-\mathrm{FET}$.

In the case of the $(25,0) \mathrm{CNT}$, Coulomb interaction enhances shot noise to superpoissonian levels, to a Fano factor of up to 1.2. It is especially interesting because, from an experimental point of view, observing $\mathrm{F}>1$ would be a real signature of this effect, since a specific mechanism is required to push $\mathrm{F}$ above 1 .

Let us also stress that if we did not consider Coulomb interaction (red diamonds), we would underestimate noise by up to about $50 \%$. The whole shaded area in Fig. 2a predicts a shot noise enhancement due to Coulomb interaction [15]. This intriguing behavior has yet to be observed experimentally.

Shot noise enhancement in the $(25,0)$ CNT-FET can be explained with the help of Fig. $3 . E_{\mathrm{C}}$ and $E_{\mathrm{V}}$ are the conduction and valence band edge profiles in the channel, respectively, whereas $E_{\mathrm{CS}}\left(E_{\mathrm{CD}}\right)$ is the conduction band edge at the source (drain), and $E_{\mathrm{BS}}$ is the energy level of the quasibound state in the valence band. When the drain Fermi level $E_{\mathrm{FD}}$ roughly aligns with $E_{\mathrm{BS}}$, holes in the conduction band in correspondence of the drain can tunnel into the bound state shifting downwards $E_{\mathrm{C}}$ in the channel. As a result, thermionic electrons injected from the source can more easily overcome the barrier. Instead, when a hole leaves the bound state, the barrier increases by the same amount, reducing thermionic injection. The noise enhancement is fully due to current modulation due to trapping/detrapping of holes in the bound state. This process leads to a random modulation of electrostatic potential in the channel, thereby modulating the barrier for electrons and therefore the thermionic electron current. In this way, noise is enhanced.

For a $(13,0)$ CNT channel, which is very close to an 
$(8,0)$ GNR channel, the energy gap is $0.75 \mathrm{eV}$. As we can see in the picture, the inclusion of Coulomb repulsion between electrons (black symbols) leads to a significantly suppressed noise. Landauer-Buttiker formula for noise only include Pauli exclusion (i.e. red symbols), and therefore would yield an overestimation of noise of up to $180 \%$ at large overdrive. From an intuitive point of view, one can see that Coulomb repulsion introduces correlations between electrons that reduce current fluctuations. Since $(13,0)$ CNTs have a much wider gap $E_{\text {gap }}=0.75 \mathrm{eV}, E_{\mathrm{V}}$ in the channel is always below $E_{\mathrm{CD}}$ in the drain, and hole injection is completely inhibited, as well as noise enhancement.
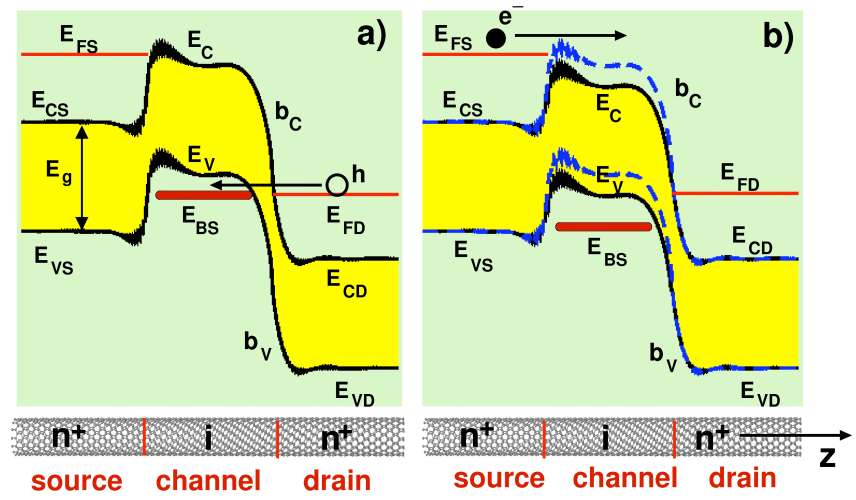

Figure 3. If an excess hole tunnels from the drain into a bound state in the intrinsic channel (a), the conduction band $E_{\mathrm{C}}$ and valence band $E_{\mathrm{V}}$ edge profiles are shifted downwards and more thermionic electrons can be injected in the channel, enhancing current fluctuations (b).
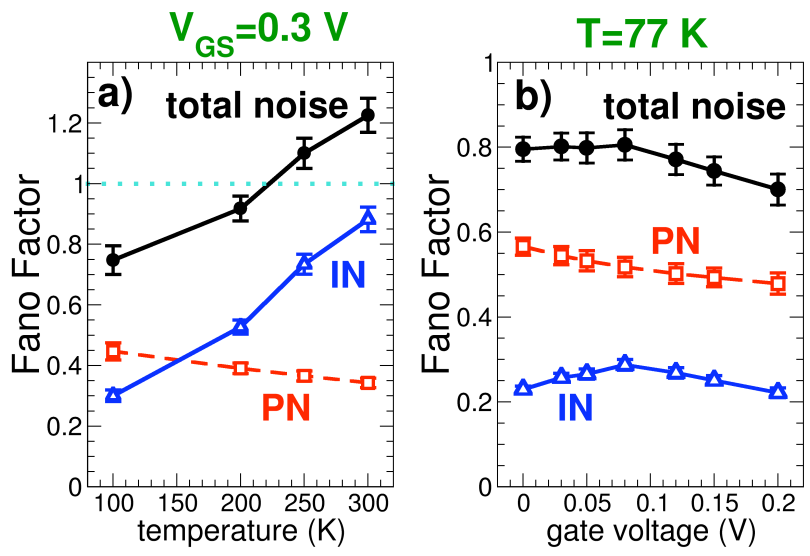

Figure 4. a) Fano factor and its two components (partition noise [PN] and injection noise [IN]) as a function of temperature. b) Fano factor at 77 as a function of gate bias, showing that superpoissonian shot noise cannot be observed at low temperature.

We have encouraged experimentalists to look at this effect in detail, because there is a clear signature of Fano factor larger than one. We also observed that the signature disappears reducing the temperature below $\sim 200 \mathrm{~K}$ (see Fig. 4), therefore room temperature measurement would be preferable to unambiguously reveal the effect.

\section{CONCLUSION}

In this paper we have discussed the shot noise properties of carbon-based transistors in which the channel laterally confined, either in the form of graphene nanoribbons or of carbon nanotubes. We show that interband tunneling can play a significant role in enhancing shot noise due to exchange of holes between drain and channel, that is a peculiar effect observable in the case of channel materials with very small energy gaps. In addition, we have shown with a simple compact model and with statistical detailed physical simulations that electron-electron interaction can lead to a significant suppression of shot noise, often overlooked when the device is described with the Landauer-Buttiker formalism.

\section{ACKNOWLEDGMENTS}

Authors gratefully acknowledge support from the European Community through the grants GRAND (Contract 215752) and NANOSIL (Contract 216171), awarded to IU.NET.

\section{REFERENCES}

[1] S. J. Tans, A. R. M. Verschueren, C. Dekker, "Room-temperature transistor based on a single carbon nanotube", Nature Vol. 393, pp. 49-52, 1998.

[2] K. I. Bolotin et al. "Temperature-dependent transport in suspended graphene". Phys. Rev. Lett., Vol. 101 art. 096802 1-4, 2008.

[3] J Tworzydło, B Trauzettel, M Titov, A Rycerz, C Beenakker, "Subpoissonian shot noise in graphene", Phys. Rev. Lett. Vol. 96, art. 246802 $1-4,2006$.

[4] R. Danneau et al. "Shot noise in ballistic graphene", Phys. Rev. Lett. Vol. 100, art. 196802 1-4, 2008.

[5] L. Dicarlo, J. Williams, Y. Zhang, D. Mcclure, C. Marcus, "Shot noise in graphene", Phys. Rev. Lett. Vol. 100, art. 156801 1-4, 2008.

[6] Z. Chen, Y. M. Lin, M. J. Rooks, P. Avouris, "Graphene nano-ribbon electronics", Physica E: Low-dim. Sys. Nano. Vol. 40, pp. 228-232, 2007.

[7] H. Ouacha et al. "Noise properties of an individual and two crossing multiwalled carbon nanotubes", Appl. Phys. Lett. Vol. 80, pp. 10551057, 2002.

[8] F. Wu et al., "Shot noise of a multiwalled carbon nanotube field effect transistor", Phys. Rev. B, Vol. 75, art. 125419 1-5, 2007.

[9] G. Iannaccone, "Analytical and Numerical Investigation of Noise in Nanoscale Ballistic Field Effect Transistors, Journal of Computational Electronics", Vol. 3, pp. 199-202, 2005.

[10]Code and Documentation can be found at the URL: http://www.nanohub.org.tools/vides.

[11] A. Betti, G. Fiori, G. Iannaccone, " Shot Noise Suppression in QuasiOne-Dimensional Field-Effect Transistors " IEEE Trans. Electron Devices, Vol. 56, p. 2137-2143, 2009.

[12]A. Betti, G. Fiori, G. Iannaccone, " Statistical Theory of shot noise in quasi-one-dimensional field-effect transistors in the presence of electronelectron interaction "Phys. Rev. B., Vol. 81, art. 035329, 2010.

[13] M. Büttiker, "Scattering theory of current and intensity noise correlations in conductors and wave guides," Phys. Rev. B, Condens. Matter, vol. 46, pp. 12 485-12 507, 1992.

[14]T. Martin and R. Landauer, "Wave-packet approach to noise in multichannel mesoscopic systems," Phys. Rev. B, Condens. Matter, vol. 45, no. 4, pp. 1742-1755, Jan. 1992.

[15]A. Betti, G. Fiori, G. Iannaccone, "Enhanced shot noise in carbon nanotube field-effect transistors", Appl. Phys. Lett., Vol. 95, art. 252108 $1-3,2009$.

[16]A. Betti, G. Fiori, G. Iannaccone "Shot noise in quasi one-dimensional FETs," in IEDM Tech. Dig., 2008, pp. 185-188. 